\title{
Localization studies in patients with primary hyperparathyroidism
}

Primary hyperparathyroidism (HPT) is currently the most common cause of hypercalcaemia in ambulatory patients and is second only to cancer-related hypercalcaemia in hospitalized patients. Approximately 1 in every 1000 adults have HPT and the incidence is even higher in selected population groups such as elderly women in which 1 in 200 have been found to have this disease. The apparent increase in HPT is not due to an epidemic but rather to the ability to make the diagnosis, particularly in many asymptomatic patients. The widespread use of auto-analysers which included serum calcium levels, was the real factor that exposed the high incidence of this disease in the ambulatory patients. In current series, anywhere from 10 per cent to 50 percent of patients are considered to be 'asymptomatic'. Nevertheless, there is a developing consensus that such patients should be operated upon unless there is a compelling medical contra-indication, because there is no way of predicting who will develop complications. Furthermore, there is increasing evidence that whereas parathyroidectomy prevents complications, it does not necessarily cure them once they are established.

There has been considerable debate about the utilization of localization studies, once the diagnosis is firmly established by biochemical testing, in patients who have not been previously explored. This has intensified with the development of noninvasive studies such as ultrasonography, technetium-thallium scintigraphy, CT and MRI scanning. It is argued that the demonstration of a suspected enlarged parathyroid by these techniques confirms the diagnosis and reassures the patient, referring physician, and surgeon that the diseased gland(s) will be found and appropriately treated. A few experienced surgeons also believe that a unilateral cervical exploration is appropriate when a single enlarged gland is found at operation in addition to an ipsilateral normal gland. Although this opinion does not represent the majority view, it is an argument used in justification for pre-operative localization in patients about to undergo their first operation. It is true that the sensitivity of localization studies has improved and they are of great value when positive in planning reoperative parathyroid explorations in which anatomical changes from a previous exploration markedly increase the difficulty and the potential morbidity ${ }^{1-3}$. Nevertheless, localization techniques can be misleading and none alone, or in combination, compares favourably with results obtained by an experienced parathyroid surgeon carrying out a primary exploration.

At best, localization procedures will demonstrate or localize the diseased gland in approximately 80 per cent of cases. Their reliability is far less in patients with hyperplasia or multiple gland disease and in those with adenomas smaller than $1 \mathrm{~cm}$ in diameter. They are particularly likely to give false negative findings in patients who have asymptomatic or uncomplicated disease or in patients with kidney stones whose calcium levels may be barely above the upper range of normal. False positive studies can also mislead or misdirect the inexperienced surgeon: as an example, thallium scans may be positive in patients with a variety of thyroid lesions including medullary carcinoma and some differentiated carcinomas as well as microfollicular adenoma. The routine use of these studies in primary cases merits critical appraisal.

High-resolution ultrasonography has been found to have good specificity (95 per cent) and a sensitivity of approximately 75-80 per cent for adenomas larger than $1 \mathrm{~cm}$. It is non-invasive, readily available and free of radiation. Its limitations are related to the location of adenomas; those in the retrotracheal and retro-oesophageal areas as well as in the mediastinum are usually not detected. Thyroid abnormalities, particularly multinodular goitres, decrease the sensitivity and specificity of the test. The specificity when positive approaches 95 per cent. Ultrasonography is best in demonstrating adenomas in juxtaposition to the lower pole of the thyroid gland. However, these are the easiest for the surgeon to detect at operation.

Thallium-technetium scintigraphy has a sensitivity of approximately 75 per cent and specificity of 90 per cent in primary cases. Again, thyroid abnormalities decrease the sensitivity and specificity and posteriorly located glands can be missed, regardless 
of size. However, some mediastinal adenomas can be detected, as well as some small cervical adenomas. A potential pitfall is that in some patients with hyperplasia or 'double adenomas', only one enlarged gland has been detected ${ }^{4}$.

CT scanning has fewer advocates in primary cases because of its cost and its sensitivity of about 75 percent. It does have the advantage of visualizing the retrotracheal and retro-oesophageal areas as well as the mediastinum. There are virtually no advocates of selective venous parathyroid hormone sampling or arteriography in the localization of parathyroid glands before a primary exploration because of their expense, invasive nature and lack of sensitivity. MRI has been found to be only 75 per cent sensitive and is also expensive.

It should be noted that most reports on the sensitivity and specificity of localization studies have come from university hospitals performing large numbers of parathyroid explorations. None, however, specifically addresses the issue of whether the experienced surgeon doing the operation was actually helped by the particular study. In as much as the experienced parathyroid surgeon is successful in finding the diseased gland(s) in 92-98 per cent of all cases, it appears unlikely that these localization studies are of any significant benefit ${ }^{4}$.

Although many patients without previous neck explorations have been referred to our clinic in recent years with ultrasound scans and scintiscan in hand, there have been no cases in which the diseased gland(s) would not have been detected without this information. Furthermore, we have been referred a number of patients for reoperative procedures who have persistent hypercalcaemia because an inexperienced surgeon proceeded with an exploration because of a positive scan. In these cases, the surgeon did not find the diseased gland, or excised only one enlarged gland in a patient with hyperplasia or 'double adenoma'. In our opinion, all patients should have bilateral neck explorations and an attempt to identify at least four glands because of the possibilities of 'double adenomas' and/or asymmetrical parathyroid hyperplasia. In evaluating patients with primary HPT who were 65 years of age or older, we found that 9 per cent had 'double adenomas'. A unilateral exploration would have failed to correct the hypercalcaemia in 5 per cent of this group. This experience reinforced our opinion that extending the operation for an average of $15 \mathrm{~min}$ to rule out the presence of an enlarged gland on the contralateral side was more than justified, even in the elderly ${ }^{5}$.

Localization studies will continue to be important in patients with persistent or recurrent hyperparathyroidism. When positive, there is no question as to their value in improving reoperative surgical results. However, until a technique has been developed with a sensitivity approaching 100 per cent, their routine use before primary neck explorations is unlikely to improve results, and may do the opposite if inexperienced surgeons are encouraged to undertake the exploration. Dr John Doppman, one of the world's most experienced authorities in evaluating parathyroid localization procedures states: 'In my opinion, the only localizing study indicated in a patient with untreated primary HPT is to localize an experienced parathyroid surgeon' ${ }^{2}$. I am in total agreement. A careful search for all parathyroid glands based on sound embryological and anatomical knowledge, and an understanding and familiarity with their pathological conditions, remain fundamental to the success of parathyroid surgery.

Division of Endocrine Surgery

N. W. Thompson University of Michigan

Michigan

USA

1. Miller D. Localization of parathyroid adenomas in patients who have undergone surgery. Radiology 1987; 162: 133-7.

2. Doppman JL. Reoperative parathyroid surgery: Localization procedures. Parathryoid Surgery. Prog Surg 1986; 18: 117-32.

3. Harness JK. Parathyroid localization studies: Are they useful? In: Thompson NW, Vinik AI, eds. Endocrine Surgery Update.
New York: Grune and Stratton, 1983 . 351-63.

4. Thompson NW, Eckhauser FE, Harness JK. The anatomy of primary hyperparathyroidism. Surgery 1982; 92: 814-22.

5. Brothers TE, Thompson NW. Surgical treatment of primary hyperparathyroidism in elderly patients. Acta Chir Scand 1987; 153: $175-8$. 